\title{
Society for Translational Medicine consensus on postoperative management of EGFR-mutant lung cancer (2019 edition)
}

\author{
Wenhua Liang ${ }^{1,2}$, Kaican Cai ${ }^{3}$, Chun Chen ${ }^{4}$, Haiquan Chen ${ }^{5,6}$, Wentao Fang ${ }^{6}$, Junke Fu ${ }^{7}$, Xiangning Fu ${ }^{8}$, \\ Shugeng Gao ${ }^{9}$, Jian $\mathrm{Hu}^{10}$, Yunchao Huang ${ }^{11}$, Ganning Jiang ${ }^{12}$, Wenjie Jiao ${ }^{13}$, Shanqing $\mathrm{Li}^{14}$, Gaofeng $\mathrm{Li}^{11}$, \\ Hecheng $\mathrm{Li}^{15}$, Hui $\mathrm{Li}^{16}$, Xiaofei $\mathrm{Li}^{17}$, Naixin Liang ${ }^{14}$, Deruo Liu ${ }^{18}$, Hongxu Liu ${ }^{19}$, Jun Liu ${ }^{1,2}$, Lunxu Liu ${ }^{20}$, \\ Yang Liu ${ }^{21}$, Qingquan $\mathrm{Luo}^{22}$, Haitao $\mathrm{Ma}^{23}$, Weimin Mao ${ }^{24}$, Zhongmin Peng ${ }^{25}$, Guibin Qiao ${ }^{26}$, Guoguang Shao ${ }^{27}$, \\ Lijie $\operatorname{Tan}^{28}$, Qunyou Tan ${ }^{29}$, Qun Wang ${ }^{28}$, Changli Wang ${ }^{30}$, Qingchen Wu ${ }^{31}$, Shidong Xu ${ }^{32}$, Songtao $\mathrm{Xu}^{28}$, \\ Lin $\mathrm{Xu}^{33}$, Yue Yang ${ }^{34}$, Fenglei Yu ${ }^{35}$, Baijiang Zhang ${ }^{36}$, Lanjun Zhang ${ }^{37}$, Bo Zhao ${ }^{8}$, Xiuyi Zhi ${ }^{38}$, \\ Alessandro Brunelli ${ }^{39}$, René Horsleben Petersen ${ }^{40}$, Chia-Chuan Liu ${ }^{41}$, Biagio Ricciuti ${ }^{42}$, Giulio Metro ${ }^{42}$, \\ Alessandro Tuzi ${ }^{43}$, Matteo B. Suter ${ }^{43}$, Matthew Evison ${ }^{44}$, Nobuhiko Seki ${ }^{45}$, Shinji Sasada ${ }^{46}$, Takhiro Izumo ${ }^{47}$, \\ William Chi-Shing $\mathrm{Cho}^{48}$, Jianxing $\mathrm{He}^{1,2}$; written on behalf of National Clinical Research Center \\ for Respiratory Disease, Thoracic Surgery Branch of China International Exchange and Promotion \\ Association for Medical and Healthcare, Chinese Alliance Against Lung Cancer and AME Thoracic \\ Surgery Collaborative Group
}

${ }^{1}$ Department of Thoracic Surgery, The First Affiliated Hospital of Guangzhou Medical University, Guangzhou 510120, China; ${ }^{2}$ Guangzhou Institute of Respiratory Disease, State Key Laboratory of Respiratory Disease, National Clinical Research Center for Respiratory Disease, Guangzhou 510120, China; ${ }^{3}$ Department of Thoracic Surgery, Nanfang Hospital, Southern Medical University, Guangzhou 510515, China; ${ }^{4}$ Department of Thoracic Surgery, Fujian Medical University Union Hospital, Fuzhou 350000, China; ${ }^{5}$ Department of Thoracic Surgery, Fudan University Shanghai Cancer Center, Shanghai 200032, China; ${ }^{6}$ Department of Thoracic Surgery, Shanghai Chest Hospital, Jiao Tong University, Shanghai 200030, China; ${ }^{7}$ Department of Thoracic Surgery, First Affiliated Hospital of Xi'an Jiaotong University, Xi'an 710061, China; ${ }^{8}$ Department of Thoracic Surgery, Tongji Hospital, Tongji Medical College, Huazhong University of Science and Technology, Wuhan 430030, China; ${ }^{9}$ Department of Thoracic Surgical Oncology, Cancer Institute \& Hospital, Chinese Academy of Medical Sciences and Peking Union Medical College, National Cancer Center, Beijing 100021, China; ${ }^{10}$ Department of Thoracic Surgery, the First Affiliated Hospital of Zhejiang University School of Medicine, Hangzhou 310003, China; ${ }^{11}$ Department of Thoracic and Cardiovascular Surgery, The Third Affiliated Hospital of Kunming Medical University (Yunnan Cancer Hospital), Kunming 650118, China; ${ }^{12}$ Department of Thoracic Surgery, Shanghai Pulmonary Hospital of Tongji University, Shanghai 200433, China; ${ }^{13}$ Department of Thoracic Surgery, Affiliated Hospital of Qingdao University, Qingdao 266071, China; ${ }^{14}$ Department of Thoracic Surgery, Peking Union Medical College Hospital, Chinese Academy of Medicine, Beijing 100730 , China; ${ }^{15}$ Department of Thoracic Surgery, Ruijin Hospital, Shanghai Jiao Tong University School of Medicine, Shanghai 200025, China; ${ }^{16}$ Department of Thoracic Surgery, Beijing Chao-Yang Hospital, Beijing 100020, China; ${ }^{17}$ Department of Thoracic Surgery, Tangdu Hospital, The Fourth Military Medical University, Xi'an 710032, China; ${ }^{18}$ Department of Thoracic Surgery, China-Japan Friendship Hospital, Beijing 100029, China; ${ }^{19}$ Department of Thoracic Surgery, Cancer Hospital of China Medical University, Liaoning Cancer Hospital \& Institute, Shenyang 110042 , China; ${ }^{20}$ Department of Cardiovascular and Thoracic Surgery, West China Hospital, Sichuan University, Chengdu 610041, China; ${ }^{21}$ Department of Thoracic Surgery, The PLA General Hospital, Beijing 100853, China; ${ }^{22}$ Shanghai Lung Tumor Clinical Medical Center, Shanghai 200030, China; ${ }^{23}$ Department of Thoracic Surgery, The First Affiliated Hospital of Soochow University, Suzhou 215006, China; ${ }^{24}$ Department of Thoracic Surgery, Zhejiang Cancer Hospital, Hangzhou 310022, China; ${ }^{25}$ Department of Thoracic Surgery, Shandong Provincial Hospital, Jinan 250021, China; ${ }^{26}$ Department of Thoracic Surgery, Guangzhou General Hospital of Guangzhou Military Area Command, Guangzhou 510010, China; ${ }^{27}$ Department of Thoracic Surgery, The First Hospital of Jilin University, Changchun 130021, China; ${ }^{28}$ Department of Thoracic Surgery, Shanghai Zhongshan Hospital of Fudan University, Shanghai 200032, China; ${ }^{29}$ Department of Thoracic Surgery, Daping Hospital, Army Medical University, Chongqing 400042, China; ${ }^{30}$ Tianjin Cancer Hospital, Tianjin Medical University, Tianjin 300060, China; ${ }^{31}$ Department of Cardiothoracic Surgery, The First Affiliated Hospital of Chongqing Medical University, Chongqing 400016, China; ${ }^{32}$ Department of Thoracic surgery, Harbin Medical University Cancer Hospital, Harbin 150040, China; ${ }^{33}$ Department of Thoracic Surgery, Nanjing Medical University Affiliated Cancer Hospital, Jiangsu Key Laboratory of Molecular and Translational Cancer Research, Cancer Institute of Jiangsu Province, Nanjing 210009, China; ${ }^{34}$ Department of Thoracic Surgery, Beijing Cancer Hospital, Beijing 100142, China; ${ }^{35}$ Department of Cardiovascular Surgery, Second Xiangya Hospital of Central South University, Changsha 410011, China; ${ }^{36}$ Department of Thoracic Surgery, Shandong Cancer Hospital and Institute, Jinan 250117, China; ${ }^{37}$ Department of Thoracic Surgery, Sun 
Yat-sen University Cancer Center, Guangzhou 510060, China; ${ }^{38}$ Department of Thoracic Surgery, Xuanwu Hospital of Capital Medical University, Beijing 100053, China; ${ }^{39}$ Department of Thoracic Surgery, St. James's University Hospital, Leeds, UK; ${ }^{40}$ Department of Cardiothoracic Surgery, Copenhagen University, Rigshospitalet, Copenhagen, Denmark; ${ }^{41}$ Division of Thoracic Surgery, Department of Surgery, Sun Yat-Sen Cancer Center, Taipei, Taiwan, China; ${ }^{42}$ Medical Oncology, Santa Maria della Misericordia Hospital, Azienda Ospedaliera di Perugia, Perugia, Italy; ${ }^{43}$ Medical Oncology, ASST Sette Laghi, Varese, Italy; ${ }^{44}$ North West Lung Centre, University Hospital of South Manchester, Wythenshawe, UK; ${ }^{45}$ Division of Medical Oncology, Department of Internal Medicine, Teikyo University School of Medicine, Itabashi-ku, Tokyo, Japan; ${ }^{46}$ Department of Respiratory Medicine, Tokyo Saiseikai Central Hospital, Minato-ku, Tokyo, Japan; ${ }^{47}$ Department of Respiratory Medicine, Japanese Red Cross Medical Center, Shibuya-ku, Tokyo, Japan; ${ }^{48}$ Department of Clinical Oncology, Queen Elizabeth Hospital, Hong Kong, China

Correspondence to: Jianxing He, MD, PhD. Department of Thoracic Surgery and Oncology, The First Affiliated Hospital of Guangzhou Medical University, Guangzhou 510120, China; China State Key Laboratory of Respiratory Disease \& National Clinical Research Center for Respiratory Disease, No. 151, Yanjiang Rd., Guangzhou 510120, China. Email: drjianxing.he@gmail.com.

Submitted Dec 03, 2019. Accepted for publication Dec 09, 2019.

doi: $10.21037 /$ tlcr.2019.12.14

View this article at: http://dx.doi.org/10.21037/tlcr.2019.12.14

\section{Introduction}

Non-small cell lung cancer (NSCLC) is the most common and fatal tumor worldwide, with 2.1 million new cases and 1.77 million deaths per year (1). With the wider application of examination approaches and the improvement of health awareness, higher proportions of surgically resectable early and mid-stage lung cancers have been detected. In overall, only $50 \%$ of patients have been cured after radical resection. In other cases, however, NSCLC is highly active and recurrence and/or metastasis can easily occur after surgery. In these patients, systemic therapy as a postoperative adjuvant therapy is required to eliminate or reduce residual micro-lesions to lower the risk of recurrence; meanwhile, the patients should be closely monitored to detect early recurrence. EGFR mutation is a major mutation type in lung cancer, and is seen in about $40 \%$ of lung cancer cases in Asia (2). Compared with wild types and other mutation types, EGFR-mutant NSCLC has its unique biological properties and drug susceptibilities, and thus requires specific diagnosis and treatment strategies. This expert consensus aims to review the current evidence and provide recommendations on key issues.

A consensus and guideline development panel, with its members including top thoracic surgeons and oncologists all around the world, was established to decide the methodologies, processes, levels of evidence, and related recommendations. The panel members proposed the core clinical issues in the consensus document and wrote and submitted the outlines to the panel for approval. The panel carried out a problem-oriented literature search for articles published since 1997 in Chinese and foreign databases. The level of evidence was defined using the following criteria: Categories of Evidence and Consensus, Category 1: based upon high-level evidence, there is uniform consensus that the intervention is appropriate; Category 2A: based upon lower-level evidence, there is uniform consensus that the intervention is appropriate; Category 2B: based upon lower-level evidence, there is consensus that the intervention is appropriate; Category 3: based upon any level of evidence, there is major disagreement that the intervention is appropriate. The strength of recommendations was classified as strong or weak according to the Grading of Recommendations, Assessment, Development and Evaluation (GRADE) system (3), and the recommendation statement was composed based on the real-world evidence. A "strong" recommendation generally refers to recommendations based on high-level evidence with consistency between clinical behavior and outcome expectancy; in contrast, a "weak" recommendation is typically based on lowlevel evidence with uncertainty between clinical behavior and outcome expectancy. After the first draft had been completed, all the panel members were involved in revising and finalizing this document. 
Consensus 1: detection of EGFR mutations is routinely recommended in surgically resected specimens of non-squamous NSCLC, and other driver mutations may also be detected if the conditions of hospital and patient allow (level of evidence: 2A; strength of recommendation: strong)

Since the use of an EGFR tyrosine kinase inhibitor (EGFRTKI) mainly depends on the presence of EGFR mutations, the National Comprehensive Cancer Network (NCCN) guidelines require the routine detection of EGFR mutations in patients with advanced non-squamous NSCLC (4). According to the results of several randomized controlled clinical trials including RADIANT, ADJUVANT, and EVAN study (5-7), EGFR-TKI has become one of the optional postoperative adjuvant treatments for patients with EGFR-mutant NSCLC. A clear postoperative EGFR mutation status helps to guide the choice of adjuvant therapy. Moreover, because different types of driver mutations suggest different biological behaviors, EGFR mutation status can predict the risk of postoperative recurrence (8) and the treatment failure patterns (9), which can guide the postoperative recurrence monitoring strategies and the drug selection after relapse. Therefore, for patients with non-squamous NSCLC, routine EGFR mutation testing is recommended after surgery. In addition, a certain proportion of nonsmokers with squamous cell carcinoma of the lungs also have EGFR mutations (10), which can be detected according to the actual situations.

With the progress in sequencing technology, multigenotyping has been widely used, and markers such as TP53 mutation and tumor mutation burden (TMB) have been found to be prognostic $(11,12)$. If the conditions of hospitals and patients allow, other driver mutations (including the main mutations recommended by the NCCN guidelines and other pathway mutations) may also be detected to provide comprehensive genotyping information for predicting prognosis and guiding treatment.

\section{Consensus 2: comprehensive prediction models based on clinical or molecular risk factors can be used to stratify recurrence risk (level of evidence: 2B; strength of recommendation: strong)}

The use of adjuvant therapy depends on the risk of recurrence. Currently, adjuvant therapy is recommended for stage II-IIIA NSCLC patients at a high risk of recurrence, whereas the population more likely to benefit from EGFR-TKI as adjuvant therapy are mainly patients with stage IIIA NSCLC. Although patients with stage I disease are at a low risk of recurrence, relapse still occurs in a notable proportion of patients. There is evidence that patients with high-risk stage I NSCLC may also benefit from adjuvant therapy (13). Many recurrence-related risk factors and comprehensive predictive models have been available for assisting in risk assessment (13-16) and thus informing tailored therapy.

\section{Consensus 3: for patients with EGFR mutations,} adjuvant EGFR-TKI can achieve longer diseasefree survival (DFS) compared with chemotherapy and thus can be used as one of the postoperative adjuvant treatment options for patients with stage II-IIIA EGFR-mutant NSCLC, especially for those patients at a high risk of recurrence and with poor expected tolerance to chemotherapy. For high-risk stage lb patients, EGFR-TKI is optionable (level of evidence: 1; strength of recommendation: strong)

In the ADJUVANT study, patients with completely resected stage II-IIIA EGFR-mutant NSCLC were treated with gefitinib for 2 years. Compared with the conventional NP regimen of 4 cycles, the median DFS was significantly prolonged from 18 to 28.7 months and the risk of disease recurrence decreased by $40 \%$ (HR $0.60, \mathrm{P}=0.005)$. In addition, patients in stage IIIA were observed to benefit more from the adjuvant gefitinib than those in stage II (6). In the EVAN study, patients with resected stage IIIA-N2 EGFR-mutant NSCLC were treated with erlotinib for 1 year; compared with the conventional NP regimen for 4 cycles, the DFS was significantly prolonged (7). In addition, severe adverse events are less common with EGFR-TKI in comparison to chemotherapy. Based on the above findings and some real-world studies (17), EGFRTKI can be used as one of the postoperative adjuvant treatment options for patients with stage II-IIIA EGFRmutant NSCLC, especially in patients at a high risk for relapse and a low expected tolerance to chemotherapy. According to most experts' consensus, for high-risk stage Ib patients, EGFR-TKI is optionable. Notably, overall survival data of the above studies is still awaited, and the positive studies were based on Chinese population so that further confirmation whether these results are translatable across the global community is warranted. 
Consensus 4: postoperative adjuvant therapies in patients with EGFR-mutant NSCLC may include the following modes: adjuvant chemotherapy, EGFR-TKI, and adjuvant chemotherapy plus EGFR-TKI (level of evidence: 2A; strength of recommendation: strong)

The main modes of postoperative adjuvant therapy for EGFR-mutant NSCLC include adjuvant chemotherapy, EGFR-TKI, and sequential use of adjuvant chemotherapy and EGFR-TKI (5-7). Due to the lack of strict head-tohead comparisons, all the above models are optional and clinicians may choose the most appropriate mode based on the patient's risk, physical performance, and willingness.

Consensus 5: the postoperative adjuvant EGFRTKI treatment should last at least 2 years (level of evidence: 2B; strength of recommendation: strong)

The use of EGFR-TKI as adjunctive therapy ranges between 0.5 and 3 years in the currently available studies $(5-7,18,19)$. In the ADJUVANT study, the recurrence-free survival curve showed a significant downward trend after 2 years, which might be explained by the discontinuation of TKI. In the previous adjuvant treatment of breast cancer with endocrine therapy and adjuvant imatinib for gastrointestinal stromal tumors, it was observed that the prolonged medication was associated with a better prognosis $(20,21)$. Furthermore, no evidence has shown that the postoperative use of TKI can induce T790M mutation (22). While the optimal duration of continuous EGFR-TKI use remains unclear, it is agreed that postoperative adjuvant EGFR-TKI should be used continuously for 2 years or more to reduce the risk of recurrence, during which time the drug toxicity should be managed.

\section{Consensus 6: patients with EGFR-mutant NSCLC} are at a higher risk of postoperative brain and bone metastases than non EGFR-mutants. Annual brain MRI and bone scans in addition to regular chest CT are recommended for EGFR-mutant NSCLC, and the scan frequencies can be increased in patients at a high risk for recurrence (level of evidence: 2A; strength of recommendation: strong)

Post hoc analysis in the ADJUVANT study and other studies have shown that the main postoperative recurrence patterns in EGFR-mutant NSCLC patients include brain metastasis, thoracic/pulmonary recurrence and metastasis, and bone metastasis (9). The current NCCN guidelines only recommend regular chest CT examinations, which may lead to delayed diagnosis of recurrence and miss the chance of re-treatment. It is therefore suggested that, based on the biological characteristics of EGFR mutations, annual brain MRI and bone scans on the basis of regular chest CT should be performed in EGFR-mutant NSCLC patients, and the scan frequencies can be increased in patients at a high risk for recurrence.

Consensus 7: in patients with recurrence and metastasis after surgery, the genetic testing results obtained from the surgical specimens could be referred to; alternatively, the surgical specimens stored within the past 2 years, or the re-biopsy specimens, may be used for genetic testing to confirm the gene mutation status to guide therapy. The detection of EGFR mutation by liquid biopsy can be a supplement when tissue samples are not available (level of evidence: 2B; strength of recommendation: strong)

Patients with postoperative recurrence and metastasis should preferably undergo re-biopsy, if conditions allow, to harvest the diseased tissue for genetic testing. If such specimens cannot be collected, the results of genetic testing in the surgical specimens can be used, as there is a high consistency of EGFR mutation between primary and metastatic lesions. Alternatively, the surgical specimens stored within the past 2 years can be used for genetic testing to identify the genetic mutation status. The detection of EGFR mutation by liquid biopsy based on PCR or NGS has high specificity and can be a powerful supplement when tissue samples are not available $(22,23)$.

\section{Consensus 8: in EGFR-mutant NSCLC patients} with postoperative recurrence and metastasis, EGFR-TKI (preferably osimertinib) can be a treatment choice for salvage therapy. In patients receiving adjuvant EGFR-TKI therapy after surgery, EGFR-TKI can be re-used if relapse occurs after drug discontinuation. If necessary, re-biopsy can be performed to confirm the T790M status (level of evidence: 1; strength of recommendation: strong)

EGFR-mutant NSCLC patients experiencing postoperative 
recurrence and metastasis can be treated with the first-line regimen for advanced disease. EGFR-TKI is recommended as a salvage therapy (24). The study series based on FLAURA found that osimertinib could significantly prolong the PFS and OS when compared with the first-line gefitinib or erlotinib. Therefore, osimertinib is the preferred firstline treatment $(4,25,26)$.

For patients who have used EGFR-TKI as adjuvant therapy, the SELECT study showed that the risk of developing T790M mutations was low (23). Thus, the same EGFR-TKI can still be used as salvage therapy, or the drug may be selected according to the above first-line therapy. If necessary, re-biopsy may be performed to identify the T790M status.

\section{The above consensus was reached among Chinese experts. To gather more extensive views on this issue, we also invited experts outside China to comment on several controversial questions involved in this consensus}

Question 1. Do patients with NSCLC who have undergone a radical resection need EGFR mutation profiling?

Expert opinion 1: Dr. Alessandro Brunelli

EGFR mutation profiling should be tested in all patients with NSCLC who have undergone radical resection. EGFR mutated patients may benefit of postoperative adjuvant EGFR-TKI in case of recurrence or locally advanced disease.

\section{Expert opinion 2: Dr. René Horsleben Petersen}

All patients with NSCLC who have undergone radical resection should be tested with EGFR mutation profiling in order to provide basis for EFGR-TKI treatment in case of locally advanced disease or recurrence.

\section{Expert opinion 3: Dr. Chia-Chuan Liu}

Only when further management is indicated, e.g., some risk-LVI, big tumor, solid or micro papillary type, should patients with NSCLC who have undergone a radical resection need EGFR mutation profiling. Stage I or patients refuse adjuvant TKI treatment do not needs the test.

\section{Expert opinion 4: Dr. Tony S. K. Mok}

Insufficient evidence for such. You may state that it is optional. For the possibility to have EGFR mutation in non- smoking squamous cell carcinoma, there is only data of such in stage IV disease and minimal for early resectable SCC.

\section{Expert opinion 5: Dr. Biagio Ricciuti \& Dr. Giulio Metro}

EGFR testing is recommended in all patients with newly diagnosed advanced NSCLC (4). The superiority of EGFR TKIs over standard cytotoxic chemotherapy in patients with advanced NSCLC harboring EGFR sensitizing mutations has been confirmed in several randomized phase III clinical trials. However, controlled randomized clinical trials with adjuvant EGFR TKIs have shown conflicting results regarding whether adjuvant EGFR-TKIs improves the survival of patients with resected NSCLC. Although a benefit in disease-free survival has been reported in the RADIANT, ADJUVANT and EVAN trials, the overall survival benefit could not be demonstrated. At the present time, in absence of a proven survival benefit with adjuvant EGFR TKIs, routine molecular testing for EGFR mutations in patients should not be recommended outside of clinical trials (4).

Expert opinion 6: Dr. Alessandro Tuzi \& Dr. Matteo B. Suter

Speaking for the European setting, the short answer is no. The only instance in which we feel a molecular profile should be carried out, is in a research setting.

\section{Expert opinion 7: Dr. Matthew Evison}

Yes. This consensus document recommends adjuvant EGFR-TKI as a potential treatment EGFR mutated NSCLC after surgery, so EGFR testing of resected tumours is essential to guide management decisions.

\section{Expert opinion 8: Dr. Nobuhiko Seki}

I agree the statement that "detection of EGFR mutations is routinely recommended in surgically resected specimens of non-squamous NSCLC, and other driver mutations may also be detected if the conditions of hospital and patient allow" and "Moreover, because different types of driver mutations suggest different biological behaviors, EGFR mutation status can predict the risk of postoperative recurrence (8) and the treatment failure patterns (9), which can guide the postoperative recurrence monitoring strategies and the drug selection after relapse. Therefore, for patients with non-squamous NSCLC, routine EGFR mutation testing is recommended after surgery". 
Expert opinion 9: Dr. Shinji Sasada

EGFR mutation profiling is necessary to plan treatment for relapse. The use of EGFR-TKI in postoperative adjuvant chemotherapy is not recommended by Japanese guidelines, so at present, EGFR mutation screening is not essential. However, I think it will be necessary in the future as CTONG data comes out.

\section{Expert opinion 10: Dr. Takhiro Izumo}

Yes. Necessary for investigating chemotherapy at the time of recurrence.

\section{Expert opinion 11: Dr. William Chi-Shing Cho}

The current data is not strong currently EGFR mutation status has no role to guide adjuvant treatment. However, the detection is attractive and may be more useful in the future.

\section{Question 2. Can EGFR-TKI replace chemotherapy in patients who require adjuvant therapy after resection? If yes, bow long should the adjuvant EGFR-TKI last?}

\section{Expert opinion 1: Dr. Alessandro Brunelli}

There is no head-to-head comparison between adjuvant chemotherapy alone $v s$. EGFR TKI vs chemotherapy+ EGFR-TKI. In patients showing mutated EGFR profile EGFR-TKI may replace chemotherapy especially if the patients are considered too high risk for conventional chemotherapy. The compliance rate of adjuvant chemotherapy after surgery is only $60 \%$ or less due to the burden of the lung resection. Especially in physiologically high-risk patients EGFR-TKI may replace chemotherapy increasing the compliance rate.

\section{Expert opinion 2: Dr. René Horsleben Petersen}

In patients with EGFR mutations and advanced NSCLC, first line treatment with EGFR-TKI provides better progression free survival (23). In the adjuvant setting, data from the ADJUVANT trial and EVAN trial show improvement in progression free survival in favor of EGFRTKI $(6,7)$. Based on these data adjuvant EGFR-TKI may replace adjuvant chemotherapy, particularly in patients with a low tolerance to chemotherapy. It is unknown how long the adjuvant EGFR-TKI should last, but probably as long as the disease is stable ( $>2$ years).

\section{Expert opinion 3: Dr. Chia-Chuan Liu}

Only when TKI is more effective than chemotherapy or patients who cannot receive or refuse chemotherapy, can
EGFR-TKI replace chemotherapy in patients who require adjuvant therapy after resection. Treatment duration depends on the purpose, but the dosage could be modified.

\section{Expert opinion 4: Dr. Tony S. K. Mok}

RADIANT study is actually a negative study; ADJUVANT is positive but in the subgroup analysis there is no benefit for stage II; and EVAN is a small size study on only stage IIIA. Data is too weak to give a $2 \mathrm{~A}$ and strong recommendation for ALL resectable lung cancer. Moreover, in terms of the length of TKI use, please note that less than $50 \%$ of patient can take more than 1 year of erlotinib in the RADIANT study.

\section{Expert opinion 5: Dr. Biagio Ricciuti \& Dr. Giulio Metro}

Adjuvant EGFR TKIs have been compared to standard chemotherapy in large phase III clinical trials with conflicting results. In two early studies, adjuvant gefitinib and erlotinib did not prolong the DFS in patients with early stage NSCLC $(5,27)$. However, both these studies neglected the EGFR mutation status. Differently, the EVAN and the CTONG 1,104 studies showed gefitinib and erlotinib improve the DFS in II-IIIA NSCLC harboring EGFR sensitizing mutations $(6,7)$. A recent pooled analysis showed that adjuvant EGFR-TKI therapy enhances DFS in patients with EGFR-mutant NSCLC but does not improve the OS (28). The lack of OS benefit represents a major concern for the use of adjuvant TKIs. The aim of adjuvant therapies is to eradicate microscopic residual disease and improve the OS. The evidence that EGFR TKIs might delay disease recurrence in high risk NSCLC is clinically relevant, but not enough to replace standard chemotherapy.

Differently, a pooled analysis of individual patient data from the largest 5 randomized largest trial of cisplatin-based chemotherapy in resected NSCLC suggested that adjuvant chemotherapy increases the 5 -year overall survival in stage IA to III NSCLC by $5.4 \%$, with a reduction of the risk of death by $17 \%$ in stage II-III NSCLC (29). EGFR-mutant NSCLC also seems more sensitive than EGFR-wild type NSCLC to chemotherapy or radiotherapy. Therefore, in absence of a head to head trial showing an overall survival benefit of EGFR TKIs compared with chemotherapy, platinum-based chemotherapy should represent the standard of care for EGFR-mutant NSCLC patients who require adjuvant systemic therapy after resection. Whether an EGFR-TKI with or without chemotherapy would improve clinical outcomes in patients with EGFR-mutant 
NSCLC in the adjuvant setting remains to be determined.

Eagerly awaited are also the results of the ADAURA trial, a phase III trial of osimertinib versus placebo in stage IB-IIIA NSCLC following complete tumor resection with or without chemotherapy. (NCT02511106).

Expert opinion 6: Dr. Alessandro Tuzi \& Dr. Matteo B. Suter

As stated in the previous answer, limited to Caucasian patients we feel that, despite its toxicity and its small survival benefit, chemotherapy remains the standard adjuvant treatment. TKIs therapy should not be proposed outside of clinical trials. The lack of data is even deeper when talking about therapy duration: although we feel that present evidence points to a longer duration, we think that we are several trials away from having a clear-cut indication.

\section{Expert opinion 7: Dr. Matthew Evison}

Yes. I would add that this "....in patients with resected stage III disease or with any N1/N2 involvement". I think the guideline should also say that overall survival data is still awaited. It is also important to state within the document that severe adverse events are less common with EGFR-TKI in comparison to chemotherapy as this is also an important aspect of decision making. It should also make reference that the two studies providing this evidence base (EVAN \& ADJUVANT) were both in Chinese populations and consideration should be given as to whether these results are translatable across the global community.

\section{Expert opinion 8: Dr. Nobuhiko Seki}

In terms of OS benefit, I do not think that EGFR-TKI replace chemotherapy in patients who require adjuvant therapy after resection. This is because OS benefit was observed only when adjuvant EGFR-TKI was used following chemotherapy (odds ratio $0.50, \mathrm{P}=0.003$ ), while OS benefit was not observed when adjuvant EGFR-TKI was used without chemotherapy $(\mathrm{P}=0.3)(30)$.

\section{Expert opinion 9: Dr. Shinji Sasada}

The CTONG data may be replaced in patients who cannot use cytotoxic anticancer drugs. In addition, regarding the effectiveness of postoperative adjuvant chemotherapy in EGFR mutation-positive patients, there are reports that EGFR inhibitors were better in exon 19 mutation patients than in exon 21 mutation patients (31). It may be beneficial for the patient to do in such case. I think the administration period is 2 years.
Expert opinion 10: Dr. Takhiro Izumo

Yes. We think that a treatment period of EGFR-TKI of about half a year is necessary.

\section{Expert opinion 11: Dr. William Chi-shing Cho}

The current available data is not very strong. However, replacing chemotherapy with TKI is a very attractive approach, though it is not yet proven to improve survival. The evidence so far is not conclusive though some data from a single center in China demonstrated the benefit, thus more evidence and studies are needed. For the duration, 2 years of adjuvant EGFR-TKI can be considered to use.

Question 3. Is it necessary to perform regular scanning for bone metastasis as well as brain MRIs for the detection of brain metastasis for EGFR-mutated patients?

\section{Expert opinion 1: Dr. Alessandro Brunelli}

EGFR-mutated patients have an increased risk of systemic disease and they should undergo regular ECTs for the detection of bone metastases and brain MRI for the detection of brain metastases.

\section{Expert opinion 2: Dr. René Horsleben Petersen}

Patients with EGFR mutations have a high risk of systemic recurrence. Regular brain MRI and bone scans as a supplement to chest CT seems reasonable for early detection.

\section{Expert opinion 3: Dr. Chia-Chuan Liu}

Usually brain Mets was symptomatic, it should be concerned all the time if there are any unexplained neurological symptoms, annularly brain MRI may also be not efficient and timely for detect brain Mets.

\section{Expert opinion 4: Dr. Biagio Ricciuti \& Dr. Giulio Metro}

The recommendation to screen for brain metastasis in stage I-III NSCLC varies across international guidelines. While all patients planned for curative stage III NSCLC treatment should receive brain MRI for initial staging, there is no consensus on stage I-II. NCCN guidelines suggest brain MRI in patients with stage IB-IIB who are candidate for curative surgery ( \pm adjuvant systemic therapies) while the ACCP guideline restricts it to patients with stage III/IV and symptomatic patients $(4,32)$. Whether regular brain MRI should be considered after curative treatment is unclear.

In the ADJUVANT trial, lung and brain metastases 
accounted for major proportion of recurrence, compared to other sites of metastases (9). In addition, the brain represents one of the most common sites of failure of EGFR TKIs in metastatic setting, suggesting a different biology ad EGFR mutant NSCLC compared to nononcogene addicted NSCLC. Although no data support the use of regular brain MRI after radical treatments, brain MRI can be proposed to patients with high risk stage III NSCLC.

There are no studies supporting the use of regular ECTs for the detection of bone metastases in patients with stage I-IIIA EGFR mutant NSCLC. Accordingly, all guidelines do not recommend regular ECTs in patients with resected NSCLC, regardless of EGFR mutation status. Therefore, ECTs should not be considered in this setting.

In conclusion, at the present time data on the association between EGFR-mutation status and a given pattern of recurrence are too weak in order to suggest a more intensive surveillance including either brain MRI and/or bone scans in EGFR-mutant patients with completely resected stage IIII NSCLC.

\section{Expert opinion 5: Dr. Alessandro Tuzi \& Dr. Matteo B.} Suter

Current available data do not suggest that an early detection of metastatic relapse can influence overall survival. We recommend, accordingly to European guidelines to perform regular thoracic CT scan, mainly directed to early local intervention (33). As stated for molecular profiling, economic consideration should apply as well.

\section{Expert opinion 6: Dr. Matthew Evison}

Yes, given the pattern of disease recurrence described in the published literature and additional factors, such as the prognostic benefit of EGFR +ve status in oligometastatic brain metastases, then it would seem appropriate to recommend a more intensive imaging regime for this patient group.

\section{Expert opinion 7: Dr. Nobuhiko Seki}

Annual brain MRI and bone scans in addition to regular chest CT are recommended for EGFR-mutant NSCLC, and the scan frequencies can be increased in patients at a high risk for recurrence. Furthermore, I think the level of evidence " $2 \mathrm{~A}$ " is reasonable. However, I think there is room for reconsidering the strength of recommendation "strong" in terms of OS benefit, I think the strength of recommendation "weak" might be reasonable.

\section{Expert opinion 8: Dr. Shinji Sasada}

EGFR mutation-positive lung cancer is likely to be complicated by bone and brain metastases, so we think that regular examination is necessary.

\section{Expert opinion 9: Dr. Takhiro Izumo}

Yes. We believe that regular examination is necessary.

\section{Expert opinion 10: Dr. William Chi-Shing Cho}

Surveillance by chest computed tomography (CT) has to be used cautiously due to its radiation hazard and lack of survival benefit. The results of the phase III IFCT-0302 trial assessing minimal versus CT-scan-based follow-up for completely resected NSCLC can be referred.

\section{Question 4. For those who relapse, is osimertinib a preferable option for EGFR+ patients?}

\section{Expert opinion 1: Dr. Alessandro Brunelli}

Osimertinib should be the preferred first-line treatment in patients experiencing postoperative recurrence and metastasis based on the latest evidence showing an improved OS and PFS compared to first line EGFR-TKI (gefitinib or erlotinib).

\section{Expert opinion 2: Dr. René Horsleben Petersen}

Osimertinib is a preferable option for EGFR+ patients as it targets both sensitizing EGFR mutation and the resistant exon 20 T790M mutation, especially in patients with CNS metastases.

\section{Expert opinion 3: Dr. Tony S. K. Mok}

FLAURA is for mostly patients who presented with stage IV disease but NOT patient who had recurred after resectable lung cancer. This is generalization and cannot be considered level 1 evidence.

\section{Expert opinion 4: Dr. Biagio Ricciuti \& Dr. Giulio Metro}

Osimertinib mesylate represents the preferred first-line option for patients with EGFR-mutant (del19/L858R) NSCLC who experience postoperative disease relapse. The approval of osimertinib in this setting is based on the results of the FLAURA trial in which he third generation EGFR TKIs osimertinib excelled over first generation EGFR TKIs (gefitinib and erlotinib) in terms of median PFS (18.9 versus 10.2 months, HR: 0.64 (95\% CI: 0.37-0.57), $\mathrm{P}<0.001$ ) and median OS (38.6 versus 31.8 months, HR: 0.79 (95\% CI: 
0.64-0.99), $\mathrm{P}=0.046)(26,34)$.

On this basis, osimertinib should be considered as the preferred treatment option at relapse in any case for patients with EGFR-mutant NSCLC (del19/L858R).

Expert opinion 5: Dr. Alessandro Tuzi \& Dr. Matteo B. Suter

Osimertinib, when available, should be the preferred option for metastatic, EGFR mutated, NSCLC patients, given its efficacy for progression free and overall survival (34). Also, given its low affinity for wild type EGFR osimertinib is better tolerated than first generation TKIs.

\section{Expert opinion 6: Dr. Matthew Evison}

Yes. There is high level evidence from FLAURA and osimertinib should be first line TKI.

\section{Expert opinion 7: Dr. Nobuhiko Seki}

On the basis of the FLAURA study, I think Osimertinib can be a preferable treatment choice in terms of multiple positive characteristics, including response rate, activity for brain metastasis, progression free survival, and overall survival.

\section{Expert opinion 8: Dr. Shinji Sasada}

Osimertinib has been reported to extend overall survival over first generation EGFR-TKI. I think this is the preferred choice for recurrent cases.

\section{Expert opinion 9: Dr. Takhiro Izumo}

Yes. However, at the present time, it is necessary to detect T790M by re-examination with tissue or liquid biopsy at the time of recurrence.

\section{Expert opinion 10: Dr. William Chi-Shing Cho}

Yes, osimertinib has demonstrated an overall survival benefit over upfront first- or second-generation EGFR-TKI (e.g., gefitinib, erlotinib or afatinib) (34). The use of osimertinib upfront results in higher objective response rate, longer progression free survival, longer overall survival but lower toxicities. It is preferred all patients with EGFR exon 19 del or L858R mutation in their tumor, but is more expensive than other EGFR-TKI.

\section{Acknowledgments}

We deeply thank Prof. Tony S. K. Mok and Prof. Calvin S. $\mathrm{H}$. $\mathrm{Ng}$ for their critical review and comments in revising this consensus.

\section{Footnote}

Conflicts of Interest: The authors have no conflicts of interest to declare.

Etbical Statement: The authors are accountable for all aspects of the work in ensuring that questions related to the accuracy or integrity of any part of the work are appropriately investigated and resolved.

\section{References}

1. Bray F, Ferlay J, Soerjomataram I, et al. Global cancer statistics 2018: GLOBOCAN estimates of incidence and mortality worldwide for 36 cancers in 185 countries. CA Cancer J Clin 2018;68:394-424.

2. Pirker R, Buder A, Filipits M. Osimertinib in advanced EGFR T790M-positive non-small-cell lung cancer: the clinical impact of AURA3. Transl Cancer Res 2017;6:S265-S269.

3. Guyatt GH, Oxman AD, Kunz R, et al. Going from evidence to recommendations. BMJ 2008;336:1049-51.

4. Available online: https://www.nccn.org/

5. Kelly K, Altorki NK, Eberhardt WE, et al. Adjuvant Erlotinib Versus Placebo in Patients With Stage IBIIIA Non-Small-Cell Lung Cancer (RADIANT): A Randomized, Double-Blind, Phase III Trial. J Clin Oncol 2015;33:4007-14.

6. Zhong WZ, Wang Q, Mao WM, et al. Gefitinib versus vinorelbine plus cisplatin as adjuvant treatment for stage II-IIIA (N1-N2) EGFR-mutant NSCLC (ADJUVANT/ CTONG1104): a randomised, open-label, phase 3 study. Lancet Oncol 2018;19:139-48.

7. Yue D, Xu S, Wang Q, et al. Erlotinib versus vinorelbine plus cisplatin as adjuvant therapy in Chinese patients with stage IIIA EGFR mutation-positive non-small-cell lung cancer (EVAN): a randomised, open-label, phase 2 trial. Lancet Respir Med 2018;6:863-73.

8. He Q, Xin P, Zhang M, et al. The impact of epidermal growth factor receptor mutations on the prognosis of resected non-small cell lung cancer: a meta-analysis of literatures. Transl Lung Cancer Res 2019;8:124-34.

9. Xu S, Zhong W, Zhang Y, et al. JCES 01.10 The Main Treatment Failure Pattern for Completely Resected Stage II-IIIA (N1-N2) EGFR-Mutation Positive Lung Cancer. J Thorac Oncol 2017;12:S1734. 
10. Chiu CH, Chou TY, Chiang CL, et al. Should EGFR mutations be tested in advanced lung squamous cell carcinomas to guide frontline treatment? Cancer Chemother Pharmacol 2014;74:661-5.

11. Fukuyama Y, Mitsudomi T, Sugio K, et al. K-ras and p53 mutations are an independent unfavourable prognostic indicator in patients with non-small-cell lung cancer. Br J Cancer 1997;75:1125-30.

12. Singal G, Miller PG, Agarwala V, et al. Association of Patient Characteristics and Tumor Genomics With Clinical Outcomes Among Patients With Non-Small Cell Lung Cancer Using a Clinicogenomic Database. JAMA 2019;321:1391-9.

13. Zeng Y, Mayne N, Yang CJ, et al. A Nomogram for Predicting Cancer-Specific Survival of TNM 8th Edition Stage I Non-small-cell Lung Cancer. Ann Surg Oncol 2019;26:2053-62.

14. Kratz JR, He J, Van Den Eeden SK, et al. A practical molecular assay to predict survival in resected nonsquamous, non-small-cell lung cancer: development and international validation studies. Lancet 2012;379:823-32.

15. Liang $W$, Zhang L, Jiang G, et al. Development and validation of a nomogram for predicting survival in patients with resected non-small-cell lung cancer. J Clin Oncol 2015;33:861-9.

16. Zhang Y, Zheng D, Xie J, et al. Development and Validation of Web-Based Nomograms to Precisely Predict Conditional Risk of Site-Specific Recurrence for Patients with Completely Resected Non-small Cell Lung Cancer: A Multi-institutional Study. Chest 2018;154:501-11.

17. Xie H, Wang H, Xu L, et al. Gefitinib Versus Adjuvant Chemotherapy in Patients with Stage II-IIIA Non-SmallCell Lung Cancer Harboring Positive EGFR Mutations: A Single-Center Retrospective Study. Clin Lung Cancer 2018;19:484-92.

18. Li N, Ou W, Ye X, et al. Pemetrexed-carboplatin adjuvant chemotherapy with or without gefitinib in resected stage IIIA-N2 non-small cell lung cancer harbouring EGFR mutations: a randomized, phase II study. Ann Surg Oncol 2014;21:2091-6.

19. Wu YL, Herbst RS, Mann H, et al. ADAURA: Phase III, Double-blind, Randomized Study of Osimertinib Versus Placebo in EGFR Mutation-positive Early-stage NSCLC After Complete Surgical Resection. Clin Lung Cancer 2018;19:e533-6.

20. Davies C, Pan H, Godwin J, et al. Adjuvant Tamoxifen: Longer Against Shorter (ATLAS) Collaborative Group. Long-term effects of continuing adjuvant tamoxifen to 10 years versus stopping at 5 years after diagnosis of oestrogen receptor-positive breast cancer: ATLAS, a randomised trial. Lancet 2013;381:805-16.

21. Joensuu H, Eriksson M, Sundby Hall K, et al. One vs three years of adjuvant imatinib for operable gastrointestinal stromal tumor: a randomized trial. JAMA 2012;307:1265-72.

22. $\mathrm{Xu} \mathrm{H}$, Baidoo AAH, Su S, et al. A comparison of EGFR mutation status in tissue and plasma cell-free DNA detected by ADx-ARMS in advanced lung adenocarcinoma patients. Transl Lung Cancer Res 2019;8:135-43.

23. Zhao ZR, Lin YB, Shao YW, et al. Mutation profile of resected EGFR-mutated lung adenocarcinoma by nextgeneration sequencing. Oncologist 2019;24:1368-74.

24. Pennell NA, Neal JW, Chaft JE, et al. SELECT: A Phase II Trial of Adjuvant Erlotinib in Patients With Resected Epidermal Growth Factor Receptor-Mutant Non-SmallCell Lung Cancer. J Clin Oncol 2019;37:97-104.

25. Rosell R, Carcereny E, Gervais R, et al. Erlotinib versus standard chemotherapy as first-line treatment for European patients with advanced EGFR mutationpositive non-small-cell lung cancer (EURTAC): a multicentre, open-label, randomised phase 3 trial. Lancet Oncol 2012;13:239-46.

26. Ramalingam SS, Gray JE, Ohe Y, et al. Osimertinib vs comparator EGFR-TKI as first-line treatment for EGFRm advanced NSCLC (FLAURA): Final overall survival analysis. Ann Oncol 2019;30:v851-v934.

27. Goss GD, O'Callaghan C, Lorimer I, et al. Gefitinib versus placebo in completely resected non-small-cell lung cancer: results of the NCIC CTG BR19 study. J Clin Oncol 2013;31:3320-6.

28. Raphael J, Vincent M, Boldt G, et al. Adjuvant Epidermal Growth Factor Receptor Tyrosine Kinase Inhibitors (TKIs) in Resected Non-Small Cell Lung Cancer (NSCLC): A Systematic Review and Meta-analysis. Am J Clin Oncol 2019;42:440-5.

29. Pignon JP, Tribodet H, Scagliotti GV, et al. Lung adjuvant cisplatin evaluation: a pooled analysis by the LACE Collaborative Group. J Clin Oncol 2008;26:3552-9.

30. Xie P, Tang W, Li X, et al. The role of EGFR inhibitors as adjuvant therapy for EGFR mutation positive non-small cell lung cancer. J Clin Oncol 2019;37:abstr 8508.

31. Yang W, Gao Y, Li X, et al. Postoperative survival of EGFR-TKI-targeted therapy in non-small cell lung cancer patients with EGFR 19 or 21 mutations: a retrospective study. World J Surg Oncol 2017;15:197.

32. Silvestri GA, Gonzalez AV, Jantz MA, et al. Methods 
for staging nonsmall cell lung cancer diagnosis and management of lung cancer, 3rd ed: American College of Chest Physicians evidence-based clinical practice guidelines. Chest 2013;143:e211S-50S.

33. Postmus PE, Kerr KM, Oudkerk M, et al. Early and locally advanced non-small-cell lung cancer (NSCLC): ESMO

Cite this article as: Liang W, Cai K, Chen C, Chen H, Fang W, Fu J, Fu X, Gao S, Hu J, Huang Y, Jiang G, Jiao W, Li S, Li G, Li H, Li H, Li X, Liang N, Liu D, Liu H, Liu J, Liu L, Liu Y, Luo Q, Ma H, Mao W, Peng Z, Qiao G, Shao G, Tan L, Tan Q, Wang Q, Wang C, Wu Q, Xu S, Xu S, Xu L, Yang Y, Yu F, Zhang B, Zhang L, Zhao B, Zhi X, Brunelli A, Petersen RH, Liu CC, Ricciuti B, Metro G, Tuzi A, Suter MB, Evison M, Seki N, Sasada S, Izumo T, Cho WC, He J; written on behalf of National Clinical Research Center for Respiratory Disease, Thoracic Surgery Branch of China International Exchange and Promotion Association for Medical and Healthcare, Chinese Alliance Against Lung Cancer and AME Thoracic Surgery Collaborative Group. Society for Translational Medicine consensus on postoperative management of EGFR-mutant lung cancer (2019 edition). Transl Lung Cancer Res 2019;8(6):1163-1173. doi: 10.21037/ tlcr.2019.12.14
Clinical Practice Guidelines for diagnosis, treatment and follow-up. Ann Oncol 2017;28:iv1-iv21.

34. Soria JC, Ohe Y, Vansteenkiste J, et al. Osimertinib in untreated EGFR- mutated advanced non-small-cell lung cancer. N Engl J Med 2018; 378:113-25. 